\title{
MLH1-deficient HCT116 colon tumor cells exhibit resistance to the cytostatic and cytotoxic effect of the poly(A) polymerase inhibitor cordycepin (3'-deoxyadenosine) in vitro
}

\author{
PATRICK IMESCH, ANOUK GOERENS, DANIEL FINK and ANDRÉ FEDIER \\ Department of Gynecology, University Hospital Zurich, CH-8091 Zurich, Switzerland
}

Received September 13, 2011; Accepted November 25, 2011

DOI: $10.3892 / \mathrm{ol} .2011 .504$

\begin{abstract}
Cordycepin (3'-deoxyadenosine) is an inhibitor of poly(A) polymerase (PAP), an enzyme crucial to mRNA 3'-end processing, which produces the shortening of poly(A) tails, leading to the destabilization of mRNAs. Cordycepin inhibits proliferation and induces apoptosis in tumor cells, indicating its antitumor activity. Defective 3 '-end processing is associated with hypersensitivity to UV treatment. We investigated the effects of cordycepin on proliferation and apoptosis in MLH1-deficient and MLH1-proficient HCT116 colon tumor cells. MLH1 is a DNA mismatch repair (MMR) protein involved in the processing of damaged DNA. Cells with defective MMR show resistance to certain anticancer drugs. The results showed that MLH1-deficient HCT116 cells are 2-fold less sensitive to the cytostatic effect of cordycepin, as compared to MLH1-proficient cells. This reduced sensitivity to cordycepin in MLH1-deficient cells was associated with reduced upregulation of the cell cycle inhibitor p21. MLH1-deficient cells also exhibited reduced susceptibility to apoptosis upon treatment with cordycepin, as demonstrated by the reduced PARP-1 cleavage. Our findings showed that MLH1-deficient HCT116 colon tumor cells are resistant to the cytostatic and cytotoxic effect of cordycepin, indicating a possible involvement of MMR in mRNA polyadenylation. The findings also suggest that cordycepin is not suitable to therapeutically encounter tumor cells lacking MLH1 expression.
\end{abstract}

\section{Introduction}

Polyadenylation of mRNA, also referred to as 3'-end mRNA processing, is crucial to mRNA stability and to the nuclear export of mRNA (1). The stabilization of the newly transcribed mRNA and the translation of mRNA into a protein are crucial

Correspondence to: Dr André Fedier, Department of Gynecology, University Hospital of Zurich, Frauenklinikstrasse 10, CH-8091 Zurich, Switzerland

E-mail: andre.fedier@usz.ch

Key words: apoptosis, proliferation, cordycepin, DNA mismatch repair, drug resistance, mRNA polyadenylation steps in protein synthesis in all cells. mRNA polyadenylation is a regulated process carried out in a multiprotein-complex, wherein poly(A) polymerase (PAP) is the enzyme performing the addition of multiple adenosine-monophosphates (150 to 250) to the mRNA, resulting in a poly(A) tail.

Cordycepin, the main constituent of the mycelia of Cordyceps militaris, is an adenosine analogue lacking the 3'-OH-group normally present in adenosine. Cordycepin inhibits mRNA polyadenylation presumably by causing chain termination after it has been incorporated as cordycepin-triphosphate (2). Cordycepin therefore leads to the shortening of the poly(A) tail length in a dose-dependent manner (3), confirming its PAP-inhibitor activity. Cordycepin has numerous biological activities, including inhibition of cell proliferation, activation of apoptosis, and inhibition of cell migration and invasiveness (4-8). Cordycepin has been shown to reduce tumor formation in mice (6) and has therefore been proposed as a cancer drug. In addition, PAP has been suggested as a potential therapeutic target (9) and its enzymatic activity has potential as an independent prognostic marker in primary breast cancer (10).

Polyadenylation has been proposed to be linked to the DNA damage response. The DNA damage-induced p53 protein inhibited the function of the cleavage stimulation factor (CstF), a key factor in the cleavage step of the mRNA polyadenylation reaction, and thereby blocked mRNA polyadenylation (11). Furthermore, reduced CstF levels resulted in UV-hypersensitivity and defective DNA repair $(12,13)$.

DNA mismatch repair (MMR) is a type of post-replicative, multiprotein repair machinery contributing to the maintenance of genome integrity and to the apoptotic destruction of damaged cells. Defective MMR function is not only associated with a mutator phenotype, microsatellite instability and cancer predisposition, but is also one cause of tumor chemoresistance and thus is believed to contribute to chemotherapy failure $(14,15)$. MMR-deficient tumor cells have been shown to be resistant to anticancer compounds including cisplatin, temozolomide, 6-thioguanine and certain anthracyclins (16). While certain compounds such as taxanes exhibit activity against MMR-deficient tumor cells, only a few compounds to which MMR-deficient cells are hypersensitive have been found. These compounds, however, failed to successfully advance into clinical trials. 
In the present study, we investigated whether HCT116 colon tumor cells lacking the expression of MLH1, a protein of the MMR multiprotein complex, are hypersensitive to cordycepin compared to those cells expressing the MLH1 protein.

\section{Materials and methods}

Cell lines and culture. A matched pair of cell lines, i.e., an MLH1-deficient human colorectal adenocarcinoma cell line (designated HCT116+ch2) and its MLH1-proficient counterpart (designated HCT116+ch3), was used. The cell lines were derived from the human colorectal adenocarcinoma cell line HCT116 containing a hemizygous mutation in MLH1, resulting in a truncated, non-functional protein (American Type Culture Collection; ATCC CCL 247, Manassas, VA, USA). The HCT116+ch3 sub-line is complemented with chromosome 3 carrying the wild-type gene for hMLH1 and is competent in MMR function. The HCT116+ch2 cell line, for reasons of chromosome balance, is complemented with chromosome 2 and is MMR incompetent. It is generally acknowledged that the chromosome complementation does not spoil the effects of DNA MMR on drug sensitivity, although the extent of possible effects from the introduction of an extra chromosome has yet to be adequately clarified. The presence or absence of MLH1 protein in the respective cell lines was routinely checked by immunoblotting. The two cell lines were maintained in Iscove's modified Dulbecco's medium (Invitrogen, Basel, Switzerland) supplemented with $10 \%$ heat-inactivated fetal bovine serum (Oxoid, Basel, Switzerland) and geneticin (400 $\mu \mathrm{g} / \mathrm{ml}$; Invitrogen) at $37^{\circ} \mathrm{C}$ in an atmosphere with $10 \%$ $\mathrm{CO}_{2}$ and $95 \%$ humidity. Cordycepin (3'-deoxyadenosine) was purchased (Sigma, Buchs, Switzerland) and aliquots prepared in water were stored at $-20^{\circ} \mathrm{C}$.

Drug sensitivity assay. The sensitivity of the two cell lines to cordycepin treatment was assessed by a clonogenic assay. In a typical setting, 500 cells in medium were plated onto $35 \mathrm{~mm}$ cell culture dishes, followed by the addition of cordycepin to the cultures on the following day. Drug treatment was either $24 \mathrm{~h}$ or continuous (7 days). For the 24 -h treatment, the cordycepin-containing medium was replaced by drug-free medium $24 \mathrm{~h}$ later, followed by further incubation for 6 days to allow colony formation. For continuous treatment, the cells were incubated for 7 days in the cordycepin-containing medium. Cells were then fixed with $25 \%$ acetic acid in ethanol and stained with Giemsa. Colonies of at least 50 cells were scored. Each experiment was carried out at least three times in triplicate cultures. The relative colony formation (percentage of clonogenic survival) was plotted against the drug concentrations, and the $\mathrm{IC}_{50}$ concentrations were calculated by linear extrapolation.

Cell lysates for immunoblot analysis. Protein expression in untreated or cordycepin-treated HCT116+ch2 and HCT116+ch3 colon tumor cells was assessed by immunoblot analysis (western blotting) using the respective cell lysates. The cell lysates were produced from cultures that were subconfluent at the time of analysis (avoiding undesired effects due to factors such as contact inhibition). Briefly, cells were grown to $70 \%$ confluence, treated with various concentra- tions of cordycepin for various periods of time, and lysed for immunoblot analysis performed following standard protocols. The protein concentration of cell lysates was determined by the BCA Protein Assay kit (23227; Pierce, Perbio Science, Lausanne, Switzerland). Cell lysate protein $(20 \mu \mathrm{g})$ was loaded and separated using SDS-PAGE, followed by blotting onto a polyvinylidene difluoride membrane (Amersham Biosciences, Otelfingen, Switzerland). Proteins were detected by the specific primary antibodies and the respective secondary, horseradish peroxidase-conjugated anti-mouse (M15345; Transduction Laboratories, Lexington, KY, USA) or horseradish peroxidase-conjugated anti-rabbit (7074; Cell Signaling; BioConcept, Allschwil, Switzerland) antibodies. The primary antibodies used were: p21 (2946; Cell Signaling) and PARP-1 (9542; Cell Signaling, recognizing the $116 \mathrm{kDa}$ full-length PARP-1 and the cleaved 89 kDa fragment). Rabbit anti- $\alpha / \beta$-tubulin (2148; Cell Signaling) was used as a sample loading control. Complexes were visualized by enhanced chemiluminescence (Amersham Biosciences) and autoradiography.

Statistical analysis. The mean \pm SD values were calculated (where appropriate). Statistical analysis was performed using the paired two-tailed Student's t-test. $\mathrm{P}<0.05$ was considered to indicate a statistically significant difference.

\section{Results}

Loss of MLH1 correlates with resistance to cordycepin in the clonogenic assay. We determined whether the loss of MLH1 affects the clonogenic potential of HCT116 colon tumor cells treated with cordycepin. The clonogenic assay data demonstrated that MLH1-deficient HCT116+ch2 colon tumor cells were 1.9 -fold less sensitive $(\mathrm{p}<0.01)$ compared to the MLH1-proficient HCT116+ch3 colon tumor cells to 24-h treatment with cordycepin (Fig. 1). The respective $\mathrm{IC}_{50}$ values $($ mean $\pm \mathrm{SD})$ were $137 \pm 7 \mu \mathrm{M}(\mathrm{HCT} 116+\mathrm{ch} 2)$ vs. $73 \pm 5 \mu \mathrm{M}$ (HCT116+ch3). Comparable results (data not shown) were found for the 7-day treatment $(2.0$-fold; $\mathrm{p}<0.01)$. The respective $\mathrm{IC}_{50}$ values were $140 \pm 25 \mu \mathrm{M}$ (HCT116+ch2) vs. $69 \pm 11 \mu \mathrm{M}$ (HCT116+ch3). This indicates that loss of MLH1 correlates with resistance to cordycepin.

Loss of MLH1 associates with reduced upregulation of p21 expression. We determined whether the loss of MLH1 in HCT116 colon tumor cells influences the expression of the endogenous cell cycle inhibitor p21. Immunoblot data demonstrated that a 72 -h treatment with $100 \mu \mathrm{M}$ cordycepin produced a marked upregulation of p21 in the MLH1-proficient HCT116+ch3 cells. By contrast, a respective upregulation was only observed with a cordycepin concentration as high as $300 \mu \mathrm{M}$ in the MLH1-deficient HCT116+ch2 cells (Fig. 2A). p21 was absent in the untreated controls. This result indicates that cordycepin-induced p21 upregulation is dependent on the presence of MLH1.

Loss of MLH1 associated with reduced apoptosis. We determined whether the loss of MLH1 affects the susceptibility of HCT116 colon tumor cells to cordycepin-induced apoptosis. Immunoblot data demonstrated that a 72-h cordycepin treatment produced less PARP-1 cleavage (a measure of ongoing 
A

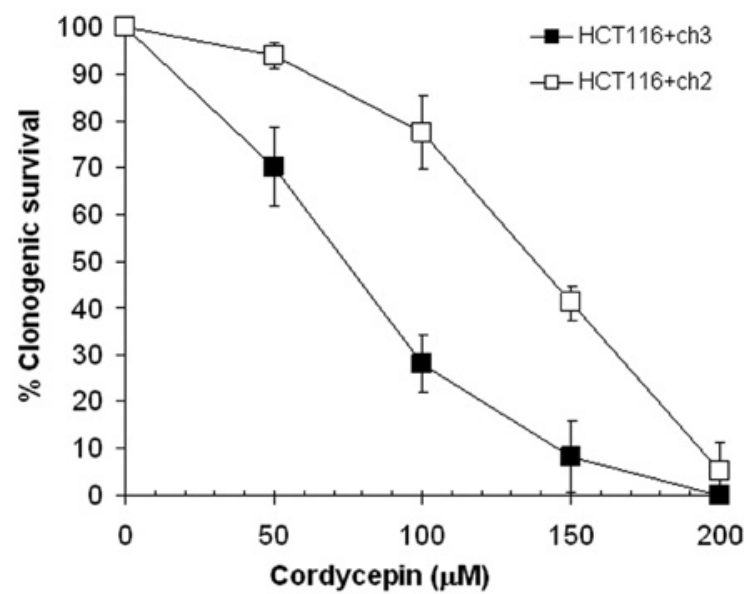

B
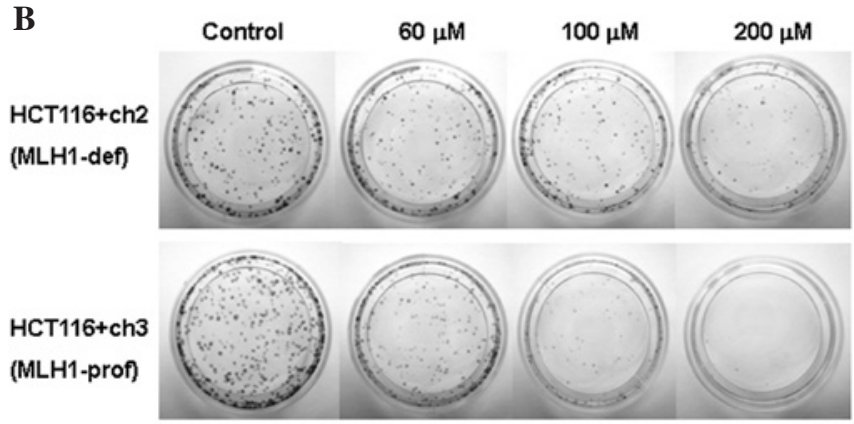

Figure 1. (A) Effect of a 24-h cordycepin treatment on the clonogenic survival of MLH1-deficient HCT116+ch2 (open squares) and MLH1-proficient HCT116+ch3 (filled squares) colon tumor cells. Cells were treated with various concentrations of cordycepin $(0,50,100,150$ and $200 \mu \mathrm{M})$ for $24 \mathrm{~h}$, followed by incubation in drug-free medium for 6 days and then by Giemsa staining. Data points are the mean \pm SD of three independent experiments performed in triplicate cultures. $\mathrm{P}<0.05$ was considered statistically significant. (B) Photographs of a representative clonogenic assay presenting colony formation as a function of cordycepin concentration. Top panel: MLH1-deficient HCT116+ch2 cells; bottom panel: MLH1-proficient HCT116+ch3 cells

apoptosis) in MLH1-deficient HCT116+ch2 cells as compared to MLH1-proficient HCT116+ch3 cells (Fig. 2B). A concentration of $300 \mu \mathrm{M}$ cordycepin was sufficient to eliminate the level of the PARP-1 precursor in MLH1-proficient cells, whereas a respective elimination was not observed with $1,000 \mu \mathrm{M}$ cordycepin in MLH1-deficient cells. The cleaved fragment of PARP-1 was hardly detectable. This inability to detect the cleaved fragment indicates that cordycepin-induced apoptosis is dependent on the presence of MLH1.

\section{Discussion}

MLH1 protein is part of the DNA mismatch repair (MMR) multiprotein complex; this post-replicative repair machinery recognizes and corrects DNA biosynthetic errors occurring spontaneously during DNA synthesis, thereby increasing replication fidelity and ensuring the maintenance of genome integrity. MMR also processes DNA damage induced by specific chemotherapeutic drugs and activates cell cycle checkpoints and apoptosis. Defective MMR, due to mutations in the MMR genes themselves or due to hypermethylation of the MLH1 gene promoter, associates with a mutator
A

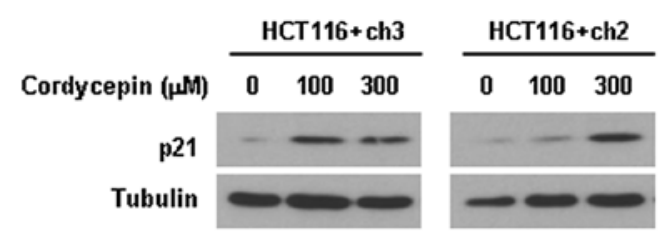

B

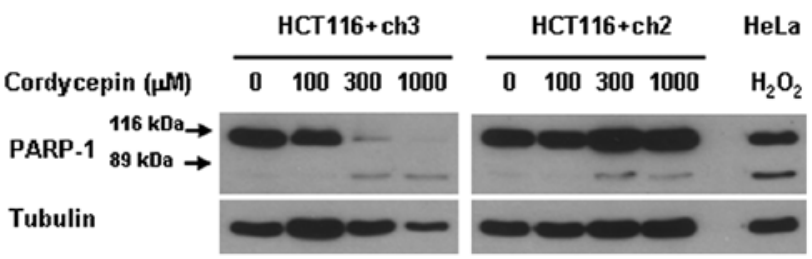

Figure 2. (A) Effect of a 72-h cordycepin treatment on the expression of p21 and (B) on the proteolytic cleavage of the $116 \mathrm{kDa}$ PARP-1 precursor into 89-kDa fragments in MLH1-deficient HCT116+ch2 and MLH1-proficient HCT116+ch 3 colon tumor cells as determined by immunoblot analysis. Cells were treated with various concentrations of cordycepin $(0,100,300$ or $1000 \mu \mathrm{M}$ ) for $72 \mathrm{~h}$, lysed, and prepared for immunoblot analysis. Tubulin is the sample loading control. HeLa cells treated with $0.8 \mathrm{mM}$ hydrogen peroxide $\left(\mathrm{H}_{2} \mathrm{O}_{2}\right)$ served as a positive control sample for apoptosis. Data are representative of two independent experiments.

phenotype, microsatellite instability, cancer predisposition and resistance to chemotherapeutically active drugs such as cisplatin and carboplatin $(14,16)$. Defective MMR predominantly associates with hereditary non-polyposis colon cancer (HNPCC), also referred to as Lynch syndrome, and, to a lesser extent, with other types cancers including those of the breast and ovaries (15). An efficient treatment of MMR-deficient cancers may require drugs to which these cancers either retain sensitivity or are even hypersensitive.

The question addressed was whether MLH1-deficient HCT116 colon tumor cells are hypersensitive to cordycepin, compared to their MLH1-proficient counterpart. Our results demonstrated that this was not the case; instead the MLH1-deficient cells were significantly less sensitive to the cordycepin-induced proliferation inhibition and apoptosis activation compared to MLH1-proficient cells. Thus, MLH1-deficient cells are associated with resistance to the PAP-inhibitor cordycepin. We suggest a role for MMR function in mediating the cytostatic and cytotoxic effects of cordycepin and therefore in mRNA polyadenylation.

The observed cordycepin resistance to proliferation inhibition in MLH1-deficient cells was the first key finding and it correlated with the delayed upregulation of p21 following cordycepin treatment, as compared to MLH1-proficient cells. p21 is an endogenous cell cycle progression inhibitor protein, which regulates cell cycle checkpoint activation and cell cycle progression attenuation, which is commonly upregulated in response to a variety of cellular stressors (17). It has been shown that the cordycepin-induced p21 upregulation and p21-dependent G2/M checkpoint activation requires the c-Jun N-terminal kinase 1 (18).

The observed apoptosis resistance in response to cordycepin treatment in MLH1-deficient cells was the second key finding and it was documented by the reduced rate of the proteolytic fragmentation of the PARP-1 full-length precursor (an event 
typical for ongoing apoptosis). Apoptosis (also referred to as programmed cell death) is important in the control of cell homeostasis in many organisms and is a crucial process in response to cellular stress $(19,20)$. Cordycepin has been shown to activate reactive oxygen species-mediated caspase-dependent apoptosis (both intrinsic and extrinsic apoptosis) in cancer cells $(21,22)$, but may also induce caspase-independent apoptosis via direct RNA synthesis inhibition (5) or via calcium- $\mu$-calpain activation (23).

Notably, absent or delayed p21 upregulation associated with resistance to proliferation inhibition and apoptosis in MLH1-deficient cells has already been demonstrated for a variety of chemotherapeutic compounds including cisplatin, carboplatin and temozolomide (16). The present study therefore adds cordycepin to this group of compounds.

Our observations indicate that the cordycepin-induced effects on proliferation and apoptosis are dependent on the presence of MLH1 protein. This finding suggests that MLH1, a protein believed to transmit the presence of MMR-dependent DNA damage to checkpoint signaling and apoptosis activation (24-26), is functionally linked to mRNA polyadenylation. However, the nature of such a potential link is unknown. MLH1 may serve either as a signal transmitter molecule or even as a detector molecule for the accumulated under-polyadenylated mRNAs. This function may then trigger proliferation attenuation and cell death signaling via JNK and p21 upregulation in the presence of MLH1, while in its absence these cellular responses do not occur.

The hypothesis that DNA damage repair and the polyadenylation mechanisms are linked was raised by recent studies. These suggest that the cleavage stimulation factor (CstF), a protein crucial for functional mRNA polyadenylation, has a direct role in the DNA damage response. Reduced CstF levels are associated with decreased viability (hypersensitivity) to UV treatment and defects of DNA damage repair $(12,13)$. Furthermore, CstF complexes with DNA damage-induced p53 protein, thereby inhibiting the function of $\mathrm{CstF}$ and finally blocking the mRNA 3' cleavage step of the polyadenylation reaction (11).

Taken together, the results demonstrate that MLH1 deficiency correlates with cordycepin resistance in colon tumor cells. Since MLH1 is one component of the MMR complex and MLH1 deficiency is associated with defective MMR function, the results have shown that MMR may be involved in managing cell responses to cordycepin treatment. Thus, cordycepin may be used in the treatment of MMR-deficient tumors.

\section{References}

1. Penman S, Rosbash M and Penman M: Messenger and heterogeneous nuclear RNA in HeLa cells: differential inhibition by cordycepin. Proc Natl Acad Sci USA 67: 1878-1885, 1970.

2. Müller WE, Seibert G, Beyer R, Breter HJ, Maidhof A and Zahn RK: Effect of cordycepin on nucleic acid metabolism in L5178Y cells and on nucleic acid-synthesizing enzyme systems. Cancer Res 37: 3824-3833, 1977.

3. Wong YY, Moon A, Duffin R, Barthet-Barateig A, Meijer HA, Clemens MJ and de Moor $\mathrm{CH}$ : Cordycepin inhibits protein synthesis and cell adhesion through effects on signal transduction. J Biol Chem 285: 2610-2621, 2010.

4. Chang W, Lim S, Song H, Song BW, Kim HJ, Cha MJ, Sung JM, Kim TW and Hwang K: Cordycepin inhibits vascular smooth muscle cell proliferation. Eur J Pharmacol 597: 64-69, 2008.
5. Chen LS, Du-Cuny L, Vethantham V, Hawke DH, Manley JL, Zhang S and Gandhi V: Chain termination and inhibition of mammalian poly(A) polymerase by modified ATP analogues. Biochem Pharmacol 79: 669-677, 2010.

6. Nakamura K, Konoha K, Yoshikawa N, Yamaguchi Y, Kagota S, Shinozuka K and Kunitomo M: Effect of cordycepin (3'-deoxyadenosine) on hematogenic lung metastatic model mice. In vivo 19: 137-141, 2005.

7. Nakamura K, Yoshikawa N, Yamaguchi Y, Kagota S, Shinozuka K and Kunitomo M: Antitumor effect of cordycepin (3'-deoxyadenosine) on mouse melanoma and lung carcinoma cells involves adenosine A3 receptor stimulation. Anticancer Res 26: 43-47, 2006.

8. Wu WC, Hsiao JR, Lian YY, Lin CY and Huang BM: The apoptotic effect of cordycepin on human OEC-M1 oral cancer cell line. Cancer Chemother Pharmacol 60: 103-111, 2007.

9. Chen LS, Stellrecht CM and Gandhi V: RNA-directed agent, cordycepin, induces cell death in multiple myeloma cells. Br J Haematol 140: 682-691, 2008.

10. Scorilas A, Talieri M, Ardavanis A, Courtis N, Dimitriadis E, Yotis J, Tsiapalis CM and Trangas T: Polyadenylate polymerase enzymatic activity in mammary tumor cytosols: a new independent prognostic marker in primary breast cancer. Cancer Res 60: 5427-5433, 2000.

11. Nazeer FI, Devany E, Mohammed S, Fonseca D, Akukwe B, Taveras $\mathrm{C}$ and Kleiman FE: p53 inhibits mRNA 3' processing through its interaction with the CstF/BARD1 complex. Oncogene 30: 3073-3083, 2011.

12. Cevher MA, Zhang X, Fernandez S, Kim S, Baquero J, Nilsson P, Lee S, Virtanen A and Kleiman FE: Nuclear deadenylation/polyadenylation factors regulate 3 ' processing in response to DNA damage. EMBO J 29: 1674-1687, 2010.

13. Mirkin N, Fonseca D, Mohammed S, Cevher MA, Manley JL and Kleiman FE: The 3' processing factor CstF functions in the DNA repair response. Nucleic Acids Res 36: 1792-1804, 2008.

14. Jiricny J: The multifaceted mismatch-repair system. Nat Rev Mol Cell Biol 7: 335-346, 2006.

15. Peltomäki P: Role of DNA mismatch repair defects in the pathogenesis of human cancer. J Clin Oncol 21: 1174-1179, 2003.

16. Fedier A and Fink D: Mutations in DNA mismatch repair genes: Implications for DNA damage signaling and drug sensitivity. Int J Oncol 24: 1039-1047, 2004.

17. Gartel AL and Radhakrishnan SK: Lost in transcription: p21 repression, mechanisms, and consequences. Cancer Res 65: 3980-3985, 2005.

18. Lee SJ, Moon GS, Jung KH, Kim WJ and Moon SK: c-Jun $\mathrm{N}$-terminal kinase 1 is required for cordycepin-mediated induction of G2/M cell-cycle arrest via p21WAF1 expression in human colon cancer cells. Food Chem Toxicol 48: 277-283, 2010.

19. Danial NN and Korsmeyer SJ: Cell death: critical control points. Cell 116: 205-219, 2004

20. Riedl SJ and Salvesen GS: The apoptosome: signalling platform of cell death. Nat Rev Mol Cell Biol 8: 405-413, 2007.

21. Jeong JW, Jin CY, Park C, Hong SH, Kim GY, Jeong YK, Lee JD, Yoo YH and Choi YH: Induction of apoptosis by cordycepin via reactive oxygen species generation in human leukemia cells. Toxicol In Vitro 25: 817-824, 2011.

22. Thomadaki H, Scorilas A, Tsiapalis CM and Havredaki M: The role of cordycepin in cancer treatment via induction or inhibition of apoptosis: implication of polyadenylation in a cell type specific manner. Cancer Chemother Pharmacol 61: 251-265, 2008.

23. Lui JC, Wong JW, Suen YK, Kwok TT, Fung KP and Kong SK: Cordycepin induced eryptosis in mouse erythrocytes through a $\mathrm{Ca}^{2+}$-dependent pathway without caspase-3 activation. Arch Toxicol 81: 859-865, 2007.

24. Nehmé A, Baskaran R, Nebel S, Fink D, Howell SB, Wang JY and Christen RD: Induction of JNK and c-Abl signalling by cisplatin and oxaliplatin in mismatch repair-proficient and -deficient cells. Br J Cancer 79: 1104-1110, 1999.

25. Wu Q and Vasquez KM: Human MLH1 protein participates in genomic damage checkpoint signaling in response to DNA interstrand crosslinks, while MSH2 functions in DNA repair. PLoS Genet 4: 1-10, 2008.

26. Yoshioka K, Yoshioka Y and Hsieh P: ATR kinase activation mediated by MutSalpha and MutLalpha in response to cytotoxic O6-methylguanine adducts. Mol Cell 22: 501-510, 2006. 